\title{
Políticas públicas y discapacidad en el Paraguay. Origen y evolución 1970 al 2006
}

\section{Public policies and disability in Paraguay. Origin and evolution 1970 to 2006}

\author{
Rosa Ruffinelli ${ }^{a}$, Stella García ${ }^{a}$
}

\begin{abstract}
Resumen
El presente trabajo tuvo como objetivo identificar el origen y evolución de las políticas públicas de discapacidad, en el Paraguay desde los años 1970 al 2006. La misma es realizada a partir de una revisión documental y posterior entrevista a referentes del área, militantes de asociaciones y organizaciones gubernamentales y no gubernamentales que trabajaron en la temática en sus inicios entre los años 1970 y 2006. El estudio fue observacional, descriptivo con enfoque cualitativo. Los principales hallazgos de la investigación tienen quever con la invisibilizarían de las personas con discapacidad, en el ámbito social, y familiar, producto de la construcción social de la misma. Otro aspecto importante de rescatar es la permanente tensión en la que se encuentran las personas y asociaciones, con relación a la visión sobre la discapacidad, considerando que conviven el enfoque de derecho, con la visión médica, lo que lleva a la comprensión sesgada de la misma, y en base a ello las acciones que proponen para el colectivo.
\end{abstract}

Palabras clave: discapacidad, políticas públicas, Estado paraguayo. a Universidad Nacional de Asunción, Facultad de Ciencias Sociales, Paraguay.

Correspondencia a: rosaruffinelli@gmail.com

Recibido:

14 junio 2018

Aceptado:

30 octubre 2018

Artículo publicado en acceso abierto bajo la Licencia Creative Commons.

(c) $(7)$

Cita:

Ruffinelli, R., \& García, S. (2018). Políticas públicas y discapacidad en el Paraguay. Origen y evolución 1970 al 2006. Kera Yvoty: reflexiones sobre la cuestión social, 3 , 29-37.

\begin{abstract}
The objective of this study was to identify the origin and evolution of public disability policies in Paraguay from the years 1970 to 2006. It is carried out based on a documentary review and subsequent interview with references of the area, activists of associations and governmental and non-governmental organizations that worked on the subject in its beginnings between 1970 and 2006.The study was observational, descriptive with a qualitative approach. The main findings of the research have to do with the invisibility of people with disabilities, in the social, and family, as a result of the social construction of the same. Another important aspect of rescuing is the permanent tension in which people and associations find themselves, in relation to the vision on disability, considering that the legal approach coexists with the medical vision, which leads to the biased understanding of the same, and based on it the actions they propose for the collective.
\end{abstract}


Keywords: disability, public politics, Paraguayan state.

\section{Introducción}

La discapacidad como construcción histórica, contextual, coyuntural situada en cada espacio geográfico, va adquiriendo de modo puntual conceptos quevan generando transformaciones en los paradigmas del modo de acercarse a ellas. Investigar temas referidos a la discapacidad nos sitúa por un lado ante personas con deficiencias en la mayoría de los casos con limitación física, mental o sensorial y por barreras que la misma sociedad les impone, que presentan en su mayoría altos grados de exclusión social, poco acceso a empleo, educación, accesibilidad a sitios públicos, entre otras desventajas sociales.

En el caso de la población en condición de discapacidad, la igualdad de oportunidades, la inclusión, la participación, aspectos estrechamente relacionados con la dignidad humana que se materializan en la libertad de las personas y en su proyecto de vida, son principios que no están garantizados efectivamente. Estas instancias que deberían ser los principales propiciadores de ello, como lo son el estado, la sociedad y la familia también están sujetas a una forma de entender y construir el concepto que generalmente surge de un discurso de normalidad.

Esta normalidad se produce en el seno del orden burgués capitalista, un orden claramente desigual y asimétrico. Al pasar de la descripción a la prescripción de un deber ser queda de manifiesto la selección arbitraria de los valores que quedan dentro de lo normal (Kipen, 2012). Esta normalidad es un dispositivo necesario para pensar la escuela, el hospital, la familia, las ciudades, dentro de patrones establecidos.

Al hablar de discapacidad, en esta investigación la asociamos a la función que tiene el Estado moderno, con relación a sus ciudadanos y ubicamos al gobierno que es el instrumento que administra los recursos estatales, que requiere de una estructura organizativa o sea de una forma de distribución jerárquica de funciones $y$ roles que posibiliten una eficiente administración.

La acción colectiva es siempre un proceso interactivo y comunicativo: implica otros actores, recursos, capacidades organizativas, habilidades de liderazgo, circunstancias coyunturales y condiciones estructurales. Esos procesos configuran escenarios en los que se hace posible o no la acción colectiva y en los que se condiciona la forma que adopta la acción, si es que existen posibilidades de existencia (Revilla Blanco, 2010). En el caso de la discapacidad estas acciones colectivas fueron desarrolladas por personas con discapacidad y sus familias, que a partir de necesidades concretas y conforme a su capacidad organizativa fueron logrando reivindicaciones para el colectivo, en un momento en que en los países latinoamericanos se producían una serie de procesos y cambios importantes que ayudaron a configurar el escenario, con reformas, revoluciones, dictaduras y nuevos procesos democráticos. Estas tensiones se tradujeron en la organización de los trabajadores para exigir derechos, la demanda por democratización de la creciente clase media, y la lucha por derechos propios de mujeres y jóvenes.

El Estado se vale de un conjunto de organizaciones especificas denominadas instituciones que se encargan de movilizar y distribuir los medios y los recursos necesarios, para prevenir, solucionar, satisfacerocontrolarsituacionesquepodrían perturbar el orden social establecido, y es través de esas organizaciones que la política pública se torna efectiva y legitima (Faleiros, 1992).

Las instituciones en su función de control político-social realizan una recuperación a través de la división de la problemática en categorías, dando como resultado el ofrecimiento de respuestas simbólicas. 
Sousa Serra (1986) toma los conceptos de Madel, y expresa que las instituciones poseen diferentes funciones: Función de formar: la misma procura moldear a los individuos, esta es una función básicamente educativa a través de un proceso ideológico que crea al mismo tiempo lo normal y lo desviado. En cuanto a la función represiva, ejerce una función también educativa, en la medida en que trae consigo la connotación del ejemplo a ser dado. Todas las instituciones tienen su lado represivo, más allá de la existencia de instituciones específicamente represivas.

Las instituciones son entonces mediaciones organizadas para convencer, amoldar y educar a través de mecanismos ideológicos convirtiendo a los servicios en mercancías y a las instituciones en vehículos de mercantilización, debido a que en la medida que una problemática determinada se constituye en amenaza de la reproducción de la fuerza de trabajo o de la paz social se crean instituciones o se llevan a cabo programas en las ya existentes.

De ahí su constitución como instrumentos para la manutención y reproducción de la hegemonía del poder central y/o instrumentos con potencialidad de alterar la hegemonía vigente.

Faleiros (1992) hace referencia a la función política de las instituciones afirmando que "las clases dominantes necesitan de canales institucionales para dar soluciones a las demandas de las clases dominadas, apaciguarlas y controlarlas", las instituciones sociales son organizaciones específicas de política social "ellas se organizan como aparatos de las clases dominantes para desarrollar y consolidar el consenso social y su hegemonía sobre los procesos sociales, y es este el principal motivo por el cual las políticas de discapacidad tuvieron una aparición tardía, a nivel nacional" (p. 87).

Según Sousa Serra (1986) "una de las maneras en que el Estado incorpora los intereses de las clases dominadas es la política social en el sentido de atender algunas reivindicaciones de los grupos subalternos y también garantizar la necesaria paz social; las instituciones son los canales de expresión de esa política" (p. 56).

Por otra parte, el concepto mismo de la discapacidad ha evolucionado con el paso de los años, tratando de definirlo más como una condición entre la persona y su entorno y no como un término que califica al individuo en sí (Sarto Martín \& Venegas Renauld, 2009) La condición física del individuo no es necesariamente la que le impide desenvolverse con facilidad dentro de sus actividades cotidianas, por el contrario, son los factores ambientales y contextuales los que causan dicha condición discapacitarte.

Aquí radica la importancia de las políticas públicas, ya que las mismas forman parte de ese entorno externo al individuo, cuyas disposiciones traducidas en normatividad pudieran generar o atenuar dicha condición (Moreno Anganita, 2010).

Si bien hasta hace muy poco tiempo la mayor parte de las investigaciones en el campo de la discapacidad ha prevalecido el enfoque individual, médico o patológico, centrándose la atención en el sujeto, en el déficit o en la alteración respecto de la norma, y sus consecuencias en el funcionamiento biológico, el desarrollo psicológico, el aprendizaje académico, la adaptación social, el desenvolvimiento en la vida cotidiana, centrando de esta forma la problemática en el ser individual de la persona y no en su entorno.

Estainvestigación apuntoaidentificar el origen y evolución de las políticas públicas de discapacidad, atendiendo que las mismas son las herramientas que permiten garantizar el verdadero ejercicio de las libertades de las personas.

\section{Material y Método}

El estudio fue observacional, descriptivo con enfoque cualitativo, a partir de una revisión documental y entrevistas, el diseño es no experimental. En una primera etapa es de tipo documental, basado en el análisis de planes de desarrollo desde 
el año 1962, hasta el año 2006, además de los instrumentos de Censos, nacionales, las leyes nacionales, y documentos e informes de las Asociaciones de personas con discapacidad, a partir del cual fueron construidas tres categorías de análisis que son: la discapacidad como construcción social, las asociaciones de padres y los organismos del estados, a partir de las características o propiedades basadas en patrones repetidos en las entrevistas y los datos documentales, por medio de la teoría fundamentada. Se optó por ella considerando que posibilita una lógica de inducción analítica ante construcciones sociales complejas emergentes, cual es el caso de la discapacidad. Fueron realizadas 7 entrevistas en profundidad a personas con discapacidad militantes tanto de organizaciones del Estado como de la sociedad civil. La muestra fue seleccionada a partir de la derivación de las personas con discapacidad hacia sus iguales. El objetivo de la investigación fue analizar el origen y evolución de las políticas de Discapacidad en el Paraguay en el periodo de 1970 al 2006. El periodo fue seleccionado considerando que en año 2006 se aprueba la Convención por los Derechos de las personas con discapacidad y con ella nuevos desafíos en la forma de ver y pensar desde las políticas públicas para las personas con discapacidad (pcd).

\section{Resultados}

La conceptualización de la discapacidad desde los paradigmas propio de las ciencias sociales es relativamente reciente (Moreno Anganita, 2010), Bourdieu sostiene que un campo es un espacio social, un microcosmos, un universo, un espacio practico de la existencia cotidiana, que de acuerdo con las leyes propias son relativamente autónomas (Bourdieu, 1992). La discapacidad como campo hace referencia a un conjunta de relaciones entendidas como las como las determinaciones que a partir de una misma lógica definen sujetos, y disposiciones o actitud, esquemas, practicas y discursos.
La discapacidad como campo entonces, promueve diversos poderes desde los sujetos, las practicas y los discursos, que pueden buscar mantener el campo con su dinámica propia, transformarlo o simplemente resistirlo. Sin embargo, estas estrategias están condicionadas por las percepciones que los sujetos tienen sobre la discapacidad como campo y que a su vez las define, da forma y contenido a sus practicas y discursos. (Torres Davila , 2004, p. 129)

La discapacidad ha sido tradicionalmente como un problema individual, que afecta a personas en particular, un problema derivado de la condición de cada una de las personas, y por lo tanto que debe ser resuelto en forma también individual y conforme a las condiciones que tienen las familias para resolver estas situaciones, en la que se ve a la persona como incompleta o desajustada con relación a lo definido como normalidad, es decir no son sujetos de protección sacial,

La protección social aparece en los documentos oficiales de Paraguay recién en el año 2004, pos Consenso de Washington y se reitera desde entonces en los distintos programas de gobierno mostrando como principal tratamiento su asimilación a los programas dirigidos a la población en Extrema Pobreza (lo que García, 2017, llama Protección Social No Contributiva). Por eso; su tratamiento respecto a la población portadora de discapacidad es de trascendental importancia, en época de instauración y necesidad de fortalecimiento de enfoques de derechos y perspectivas que involucran lo social con protagonismo, tal como en enfoque bio psico social para el diseño e implementación de las políticas públicas dirigida a personas con discapacidad.

Cabe resaltar dos grandes características que contextualizan las políticas sociales en general en Paraguay (García y García, 2017) y son; 1o.) la 
imposibilidad de separar el modelo de política social predominante en Paraguay del modelo de política económica, lo que implica que; la asistencia y la promoción social desde el Estado, con participación de otros actores como el mercado, la familia, la comunidad; etc., se corresponden históricamente al proceso productivo que la sociedad impulsó históricamente en países con esquemas más o menos similares (aunque en América Latina con menor desarrollo) a lo que en Europa se denominó Estado de Bienestar en el siglo pasado. Esto; para acompañar el proceso productivo industrial de esos países. En el caso de Paraguay el proceso de producción y acumulación al que es sometido desde su inserción al capitalismo internacional tras la guerra de la triple alianza y hasta hoy le configuraron como rol clave; ser productor primario, sin desarrollar industrias nacionales de relevancia, tal como los países de la región que pasaron por la industrialización por sustitución de importaciones en el siglo pasado, este es un dato clave para interpretar Paraguay. Lo segundo es que Paraguay vivió una de las mas largas dictaduras de A. Latina, lo que configuro un determinado tipo de Estado y Sociedad cuya relación atraviesa todas las esferas de la vida social, por tanto, traspasa toda la mirada ciudadana que se podía configurar respecto a la discapacidad. Las 2 características históricas imprimen rezago tanto en las Políticas Sociales como en la actual configuración de la Protección Social, que acaba incidiendo en los procesos para distintos grupos poblacionales, entre los que situamos a las personas con discapacidad.

En el casode Paraguay, la discapacidad se mantuvo mucho tiempo invisible a la vida pública, solo enmarcada en el ámbito doméstico, y por lo tanto asumido y resuelto por este ámbito.

mis padres se hicieron cargo de mi desde que nací, todas mis necesidades fueron proveído pro ellos, no hubo servicios de salud ni educación públicos a los que pudiera acceder, porque yo era diferente decían". "Había un desconocimiento acerca de la discapacidad, no había estadística, médicos especialistas, solo existía una escuela diferencial denominada Máximo Arellano Se desenvolvían en un ambiente bastante discriminatorio, las familias bien posicionadas económicamente negaban si en su seno familiar existía una persona con discapacidad y esto representaba un obstáculo a la hora de contar con algún dato estadístico. (E1)

Esto se evidencia en la revisión documental realizada en los planes de desarrollo de la Secretaría Técnica de Planificación del Desarrollo Económico y Social que desde su creación el 6 de marzo de 1962 (Ley № 312/62), no registra información sobre discapacidad en los planes de desarrollo nacional. Es así como fueron revisados los Planes de Desarrollo de los años 1965, 1967-1968, 1967-1981, 1985-1989, 1989-1990, y en ninguno de ellos directa o indirectamente hace mención de la necesidad de atención a las minorías o diferencias.

En cuanto a los Censos Nacionales, realizados entre los años 1962, al 2002 fueron, solo el del 1972, no se contempló preguntas vinculadas a la materia. La producción de información que permita profundizar sobre la misma es limitada, se puede señalarse que los primeros conatos de medición de la discapacidad fueron realizados en el marco del Censo Nacional de Población y Viviendas del año 1962 y posteriormente se efectuaron nuevos intentos en los censos de 1982, 1992, 2002. En el año 1962, la pregunta 20 hablaba impedimentos físicos permanentes, ciego, sordo, sordomudo, no hallándose incidencia en los resultados. En el año 1982, se obtuvo un $1.07 \%$ y la pregunta $21 \mathrm{del}$ instrumento del censo expresaba “ ¿Alguno de los miembros de su hogar es ciego, mudo, sordo o tiene algún impedimento físico u otra deficiencia? Ciego Sordo Mudo 
Paralítico u Otro, hay que considerar que para ese año ya fue creada el INPRO, como institución de protección de personas excepcionales, por lo que de alguna manera empieza a formar parte la discapacidad de la vida pública y muy tímidamente el estado reconoce la problemática. En el año 1992 el Censo Nacional registro $0.96 \%$ con la pregunta "5. ¿Tiene algún impedimento físico o mental? Si (Ciego, sordo, mudo, paralítico, otro) No y en el año 2002 con un $0.97 \%$ en respuesta a la pregunta 36 ¿Existe en este hogar alguna persona que tiene impedimento físico o mental de forma permanente? si/no.

En esta perspectiva, anclada en los desarrollos funcionalistas la discapacidad es pensada en términos de tragedia personal o de desviación social. (Rosato, 2009, p. 76)

Con esta forma dever la discapacidad, se ve a la persona como devaluada, con un valor inferior, y el ejercicio de sus derechos y por lo tanto se encuentra supeditada bajo la tutela permanente de otro, ya que la discapacidad es una condición permanente, no una enfermedad de la que puede curar, así fue vista por muchos años, como seres inferiores no pertenecientes a los círculos sociales, yen muchos casosocultos. Empieza a tomar relevancia a partir de la década del 70 , los casos en principio son atendidos por organizaciones privadas, que trabajaban exclusivamente el área de retardo mental, desde el aspecto educativo formal, todas las demás discapacidades eran consideradas y asumidas desde la intimidad del grupo familiar. Es así que nace la escuela Máximo Arellano en el año 1956, que los que plantea es dar normalidad a los anormales, es decir que también vayan a las escuelas, como los demás niños de su edad. Con el correr de los años esto trasciende al decir de al decir de Torres Davila (2004) "las relaciones que se establecen en su interior son de fuerza y de luchas, ya que cada posición y cada sujeto posee diferentes capitales" (p. 76) y es de esta forma cuando se plantea a nivel de parlamento nacional la elaboración y promulgación de una Ley que por primera vez integre a las personas con discapacidad a la vida social del país, aunque desde una perspectiva proteccionista, comoun modelo de atención de la discapacidad desde lo médico o rehabilitador, en donde lo que se busca es "curar", por lo que se propone son una serie de servicios exclusivos para estas personas.

Recién en los años 70, según relata el Dr. Bonifacio Irala Amarilla "que a partir de una situación particular con su hija, Tonia Bernardita" empieza a ver la necesidad de que las personas con discapacidad también requerían servicios y que el estado no le estaba brindando ninguna atención, es más estaba invisibilizando a esa población, a partir de esto, aunque inicialmente este requerimiento solo se da con las personas con deficiencia mental, atendiendo al reclamo de padres en cuanto a su necesidad de escolarizaciónm primordialmente", es importante remarcar que en ese momento el era Senador de la Nación, y la necesidad de legislación para las personas con discapacidad se ve a partir de la propuesta de éste, el padre de una niña con discapacidad del entorno político y económico del momento, y que como diría (Torres Davila, 2004), el capital individual de los entornos va movilizando diversas fuerzas, que configuran el campo de la discapacidad.

En este momento también empiezas las organizaciones no gubernamentales a organizarse en base a las necesidades de sus hijos con discapacidad y abre sus puestas la Fundación Solidaridad. La Fundación Solidaridad fue iniciativa del Prof. Dr. Carlos Mersán, quien trajo de San Pablo la idea de la estructura y la logística para un centro de atención a las personas con discapacidad física. En el año 1973 se crea CERENIF (Centro de Rehabilitación del Niño Impedido Físico), iniciando con consultorio de pediatría y traumatología y en 1973 - con la construcción del local en e barrio Mburukujá, En 1980 se conforma el Taller Ortopédico de Ortesis: ANDAR, el 
primero del Paraguay en su especialidad. que trabaja con niños con discapacidad física. Todas estas iniciativas según comentan los informantes surgen a partir de la búsqueda de tratamientos a personas con discapacidad de padres y familiares de niños y niñas de clases sociales más favorecidas, y como tal centra su prestación de servicios en esas poblaciones.

Es importante destacar que este tipo de servicios solo se contaba en la capital del país, por lo que su nivel de cobertura era relativamente bajo para los índices establecidos a nivel mundial, que hablaban de un 10 al $15 \%$ de la población total, y con mayor incidencia en los países más pobres.

Cuando yo nací mis padres vivían en el interior, y mi mamá le dijo a mi papá vamos a Asunción, porque esta criatura tiene algo, y aquí no le van a curar, allá hay mas doctores. (E7)

Es importante remarcar que inicialmente si bien el estado no tomaba en cuenta a las personas con discapacidad como beneficiarios de sus políticas públicas, tampoco lo hacia la familia. La persona con discapacidad por su condición misma no era considerada sujeto de derecho, era invisible a toda la sociedad.

No era el dictador nomás el que no nos veía como personas, con derechos era toda la sociedad ni siquiera éramos vistos en nuestra individualidad, ni sexualidad, ni siquiera era hombre, éramos simplemente invisibles. (E5)

nunca sentí la necesidad del estado, porque mi familia me brindo todo, pero si es cierto que si necesitaba tampoco posiblemente encontraría nada en los servicios publicos. (E3)

El 12 de septiembre de 1979 llegó a manos del entonces Presidente de la Cámara de Diputados, J. Augusto Saldívar el pedido formal de creación de una institución que se abocara a las Personas con Discapacidad, ya que hasta ese entonces las leyes no contemplaban tal posibilidad. La elaboración de la propuesta de la ley.

El impulsor de la legislación, el doctor Bonifacio Irala Amarilla planteó la necesidad, argumentando que ya en ese entonces y según datos de la Organización Mundial de la Salud (OMS) por lo menos 5 de cada 100 personas a nivel mundial padecían de algún tipo de discapacidad y que Paraguay formaba parte de esas estadísticas.

Es así que el 30 de noviembre de 1979 se promulga la Ley número 780 que crea el Instituto Nacional de Protección a Personas Excepcionales (INPRO), dependiente del Ministerio de Educación y Culto, designándose a Alejandrina Rodríguez Duarte de profesión abogada, como la primera directora. Este nivel de dependencia la posiciona como subalterna como institución con una lectura subordinada de su función, y con una mujer como responsable, lo que nuevamente relega el rol de cuidado a la mujer, a la ubica en una posición de Institución Inferior, en relación a las demás Organizaciones del Estado.

La ley se orientaba esencialmente a prestar asistencia y a la rehabilitación física, pero dentro de un esquema médico, asistencial, en donde seguían las personas con discapacidad como objetos de la intervención estatal.

En el año 1983, con el objetivo "de capitalizar experiencia de otros países que puedan ser aplicadas al campo nacional, y recibir los últimos conocimientos en técnicas que sirvan para acciones futuras" (I Seminario Interamericano sobre la rehabilitacion de las personas excepcionales, 1983), en donde se ve sigue centrado el proceso a los aspectos médicos de la discapacidad, paradigma del momento, al profesional de rehabilitación como centro de este, y a la personas con discapacidad y sus familias como meros receptores. 
Durante este primer periodo, como política social estaba tutelada por instituciones internacionales como el Instituto Interamericano del Niño, que y lo que se busco prestar asistencia a las personas con discapacidad sin instalar un sistema de servicios que cubra todo el territorio nacional, es más fue acotado al área de Asunción y sus alrededores, que coincide con el periodo en donde la dictadura ejerció con mayor fuerza su capacidad destructora de la diversidad.

La dictadura nos mató culturalmente. Nos sacó la sensibilidad hacia nuestro prójimo. Todo era motivo para reprimir: si uno era homosexual, si tenía el pelo largo, si tenías barba, la discriminación validó históricamente la represión en este país. (E2)

Con el advenimiento de un proceso más democrático, se suponía que la realidad de las personas con discapacidad pudiera haberse modificado, pero no fue así, las organizaciones existentes comenzaron a buscar ingresos para el financiamiento de acciones, siempre tendientes a la rehabilitación, considerando el déficit con que se contaba en esta área, aun no enfocada en los derechos, sino más bien al cuidado de la salud y la rehabilitación.

La democracia no quito el enemigo común, y entonces nos sentimos un poco pedidos, nos dividimos y con ello nos debilitamos. (E4)

En el año 1991 se presenta la propuesta de "Plan de actuación Integral para personas con discapacidad, 19921995, cuyo patrocinio estuvo a cargo del Programa de las Naciones Unidas, en este documento ya se hace referencia a la necesidad de estructurar el sector de atención a discapacidad (Comité Nacional de Coordinacioón sobre Discapacidades en el Paraguay, 1992).
En la revisión de los documentos de ARIFA (Asociación de Rehabilitación de impedidos físicos), se observa que la preocupación se centra en:

-Creación de un centro de rehabilitación para personas con discapacidad fecha (ARIFA, 1997).

-Discusión sobre el alcance de las acciones de INPRO en la atención de las salud y provisión de medicamentos (ARIFA, 1998).

Podríamos ubicar un segundo periodo que inicia en el 2004 con esta nueva generación de políticas públicas, por primera vez aparece la protección social en el discurso del estado, con la pero de manera limitada a los programas de reducción de la pobreza, y trasverzalizada en ella la discapacidad, pero aun sin ser considerada, como una causa de la misma.

En esta etapa se da la participación activa y permanente de las organizaciones de la sociedad civil que trabajan en el sector, pero la principal influencia hacia el estado viene de los organismos internacionales que presionan para la consolidación de instancias institucionales que rijan la temática a nivel nacional.

\section{Conclusión}

$\mathrm{Si}$ bien la discapacidad como categoría de análisis es muy incipiente y mas bien es vista desde las ciencias médicas, enarcadas en la anormalidad, esta primera aproximación visualiza que aun la visión desde las personas con discapacidad es desde la perspectiva médica, lo que refleja en las acciones y preocupaciones tanto de las personas con discapacidad, así como desde las respuestas del estado a las solicitudes de los mismos.

Las asociaciones de y para personas con discapacidad han tenido una participación sostenida a lo largo del tiempo apuntando al modelo asistencial médico, producto de las necesidades emergentes del colectivo, por lo menos en este periodo estudiado, desde los años 1970 
hasta el año 2006, lo que también responde al modelo en el cual se desenvuelve las organizaciones sociales tanto del Paraguay como del entorno americano.

El estado asumió muy tardíamente la responsabilidad sobre las personas con discapacidad, con una mirada asistencial no promocional, en base a exigencias internacionales, no surgidas de las propias instituciones.

\section{Referencias Bibliográficas}

ARIFA. (11 de o4 de 1998). Acta № 5. Asunción: Documento no publicado.

ARIFA. (22 de o9 de 1997). Acta № 9. Asunción: Documento no publicado.

Bourdieu, P. (1992). Las reglas del arte. Génesis y estructura del campo literario. Barcelona: Anagrama.

Comité Nacional de Coordinación sobre Discapacidades en el Paraguay. (1992). Documento de trabajo № 1. Asunción: documento no publicado.

Faleiros, V. (1992). Metodología e Ideología del Trabajo Social (4ta. ed.). Buenos Aires, Argentina: Humanitas.

García, C. (2017). Protección social no contributiva en Paraguay; un balance a 10 años de su implementación. Asunción: Investigación para el Desarrollo. http://209.177.156.169/ libreria_cm/archivos/pdf_78o.pdf

García, S., \& García, C. (2017). Rasgos Claves de la Protección Social en
Paraguay, Ponencia. 1er Foro Mundial del Pensamiento Crítico. Buenos Aires: CLACSO.

I Seminario Interamericano sobre la Rehabilitación de las Personas Excepcionales. (24-28 de octubre de 1983). Conclusiones y recomendaciones finales. Asunción: Documento no editado.

Kipen, E. (2012). En torno a una conceptualización -(im)posible- de la discapacidad. En M. Almeida, \& M. Angelino (Eds.), Debates y perspectivas en torno a la discapacidad en América Latina (p. 128-131). Entre Rios: Facultad de Trabajo Social UNINTER.

Moreno Anganita, M. (2010). Infancia, políticas y discapacidad. Bogotá: Universidad Nacional de Colombia.

Revilla Blanco, M. (2010). América Latina y los movimientos sociales: el presente de la «rebelión del coro». Nueva Sociedad, (227), 51-67. https://nuso.org/media/ articles/downloads/3696_1.pdf

Sarto Martín, M., \& Venegas Renauld, M. (2009). Aspectos clave de la Educación inclusiva. Salamanca: Publicaciones del INICO.

Sousa Serra, R. (1986). La Práctica Institucional del Servicio Social. Sao Paulo, Brasil: Cortez Editor.

Torres Dávila, M. (2004). Discapacidad y Genero: Mas allá del sentido de la maternidad diferente [Tesis de Maestría en Estudios de Género]. Quito: FLACSO.

\section{Sobre las Autoras}

Rosa Ruffinelli

Trabajadora Social por la Universidad Nacional de Asunción. Doctoranda en Educación por la Universidad Nacional de Asunción. Tutor de tesis de la FENOB/UNA, Docente de la Materia Abordaje Profesional y docente investigador FACSO, UNA.

Stella García

Licenciada en Trabajo Social por la Universidad Nacional de Asunicón. Magíster en Trabajo Social por la Universidad Federal de Río de Janeiro. Docente de grado y posgrado en temas referidos a políticas sociales, trabajo social e intervención social. 